\title{
Acknowledgement to Referees
}

(C) King Abdulaziz City for Science and Technology 2012

The Chief Editors and the Editorial Board thank the reviewers listed below for their contribution and timeconsuming efforts in refereeing papers submitted to 3 Biotech in 2011. Their timely response and critical comments have not only assisted us in the selection of high-quality scientific work but their critique is also appreciated by authors as it frequently assists them to improve the style and content of their publications.
Professor Dr. M.H. Abd-Alla

Dr. A. Aboussekhra

Dr. R. Adeleke

Dr. A.L. Ahmad

Professor A. Alhomida

Dr. N. Al-Khalifah

Dr. I. Al-Saleh

Dr. G. Alsbeih

Dr. A. Altawashi

Dr. S. Arora

Dr. S. Ashmore

Dr. A. Aygan

Dr. B. Bajaj

Dr. S. Bajekal

Dr. A. Bamezai

Dr. R. Banerjee

Dr. A. Best

Dr. S. Bhattacharya

Dr. P. Biely

Dr. A. Bilgrami

Dr. D. Brar

Professor C. Brey

Dr. C. Brown

Dr. J.-C. Côté

Dr. A. Chehregani

Dr. Z.-M. Chi

Dr. I. Cock
Professor V. Cody

Professor B. Connolly

Professor B. Crother

Dr. S. Dastager

Dr. S. Datta

Professor S. Dave

Dr. J. Davis

Professor B. Dijkstra

Dr. C. Dolinski

Dr. X. Du

Mrs. L. Duffy

Dr. N. Dzimiri

Dr. R.U. Ehlers

Dr. M.-L. Fardeau

Mr. J.P.L. Faria

Dr. I. Ferreira

Professor S. Garg

Dr. S. Gerdes

Dr. S. Ghosh

Mr. H. Golzary

Dr. J.M. Granjeiro

Dr. A. Gschaedle

Dr. S. Hasnain

Dr. C. Henry

Dr. E. Hitti
Dr. Y. Hong

Dr. A.A.S.A. Husaini

Dr. I. Iliev

Dr. G. Jadeja

Dr. A. Jaouani

Dr. L. Ji

Professor P. Johnston

Dr. N. Kango

Dr. D. Kennedy

Dr. K.-P. Kim

Dr. T.-J. Kim

Dr. N. Kimura

Dr. V. Kitpreechavanich

Dr. J. Klovins

Dr. L. Kong

Dr. P.N. Krishnan

Dr. A. Kumar

Dr. A. Kumar

Dr. A.A. Kwaasi

Professor N. Labrou

Dr. R. Lal

Dr. J.-H. Lee

Dr. F. Li

Dr. W. Liang

Professor P. Liu 
Dr. J. Liu

Dr. R. López-Mondéjar

Dr. C. Love

Professsor D. Madamwar

Dr. D. Maiti

Dr. A. Maszenan

Dr. T. Mechichi

Dr. K. Medicherla

Dr. M.K. Meghvansi

Dr. S.H. Mirdamadian

Professor M. Miyazaki

Professor H. Modi

Dr. D. Monies

Dr. P. Morais

Dr. T. Morohoshi

Dr. S. Moukha

Dr. Y. Nagata

Dr. S. Neelgund

Dr. D. Neeraj

Dr. V. Nigam

Dr. C. Ogugbue

Dr. B. Ollivier

Associate Professor S. Orgeig

Dr. A. Panneerselvam
Professor K. Pappa

Dr. R.S. Parhar

Dr. S. Philipsen

Dr. P. Pope

Dr. C. Prabha

Dr. H. Purohit

Dr. G. Pusch

Dr. S. Rajkumar

Dr. R. Ramamoorthy

Dr. K. Ratanakhanokchai

Dr. L. Reddy

Dr. S. Reddy

Dr. G. Sablok

Dr. K. Sakka

Professor T. Sawidis

Dr. J. Schmidberger

Dr. S. Seaver

Dr. M. Sesli

Professor R. Seviour

Dr. J. Sharma

Dr. S. Shen

Dr. P. Shukla

Dr. S. Shukla

Dr. M. Sigrist

Dr. A. Singh
Dr. R. Singh

Dr. S. Singh

Dr. O. Sinitsyna

Dr. D. Soares

Dr. E.A. Somado

Dr. P. Somvanshi

Dr. K.R. Sridhar

Professor R. Subramanian

Dr. Y. Tamaru

Dr. T.-C. Tan

Dr. R. Taylor

Dr. P. Theerakulpisut

Dr. M. Troggio

Dr. A. Viswanathan

Dr. V. Vonstein

Dr. D. Vyas

Dr. X. Wei

Dr. F. Xia

Dr. Z. Yin

Dr. W. Zheng

Dr. C. Zhu

Dr. D. Zhu 